\title{
The Lyot project: toward exoplanet imaging and spectroscopy
}

Ben R. Oppenheimer, Andrew P. Digby, Laura Newburgh, Douglas Brenner, Michael Shara, et al.

Ben R. Oppenheimer, Andrew P. Digby, Laura Newburgh, Douglas Brenner, Michael Shara, Jacob Mey, Charles Mandeville, Russell B. Makidon, Anand Sivaramakrishnan, Remi Soummer, James R. Graham, Paul Kalas, Marshall D. Perrin, Lewis C. Roberts, Jeffrey R. Kuhn, Kathryn Whitman, James P. Lloyd, "The Lyot project: toward exoplanet imaging and spectroscopy," Proc. SPIE 5490, Advancements in Adaptive Optics, (25 October 2004); doi: $10.1117 / 12.552211$

EPIE Event: SPIE Astronomical Telescopes + Instrumentation, 2004, Glasgow, United Kingdom 


\title{
The Lyot Project: Toward Exoplanet Imaging and Spectroscopy
}

\author{
Ben R. Oppenheimer ${ }^{* a}$, Andrew P. Digby ${ }^{\mathrm{a}}$, Laura Newburgh ${ }^{\mathrm{a}}$, Douglas Brenner ${ }^{\mathrm{a}}$, Michael Shara ${ }^{\mathrm{a}}$, \\ Jacob Mey $^{\mathrm{a}}$, Charles Mandevillea, Russell B. Makidon ${ }^{\mathrm{b}}$, Anand Sivaramakrishnan ${ }^{\mathrm{b}}$, Remi \\ Soummer ${ }^{b}$, James R. Graham ${ }^{c}$, Paul Kalas ${ }^{c}$, Marshall Perrin ${ }^{\mathrm{c}}$, Lewis C. Roberts, Jr. ${ }^{\mathrm{d}}$, Jeffrey Kuhn ${ }^{\mathrm{e}}$, \\ Kathryn Whitman ${ }^{\mathrm{e}}$, James P. Lloyd ${ }^{\mathrm{f}}$ \\ ${ }^{a}$ Division of Physical Sciences, American Museum of Natural History, 79 St. at Central Park West, \\ New York, NY, USA 10024 \\ ${ }^{\mathrm{b}}$ Space Telescope Science Institute, 3700 San Martin Blvd., Baltimore, MD, USA 21218 \\ ${ }^{c}$ Department of Astronomy, University of California-Berkeley, Berkeley, CA, USA 94720-3411 \\ ${ }^{\mathrm{d}}$ AMOS - The Boeing Company, 535 Lipoa Pkwy Suite 200, Kihei, HI, USA 96753 \\ eInstitute for Astronomy, University of Hawaii, 2680 Woodlawn Dr., Honolulu, HI, USA 96822 \\ ${ }^{\mathrm{f}}$ California Institute of Technology, 105-24, Pasadena, CA, USA 90025
}

\begin{abstract}
Among the adaptive optics systems available to astronomers, the US Air Force Advanced Electro-Optical System (AEOS) is unique because it delivers very high order wave front correction. The Lyot Project includes the construction and installation of the world's first diffraction-limited, optimized coronagraph that exploits the full astronomical potential of AEOS and represents a critical step toward the long-term goal of directly imaging and studying extrasolar planets (a.k.a. "exoplanets"). We provide an update on the Project, whose coronagraph saw first light in March 2004. The coronagraph is operating at least as well as predicted by simulations, and a survey of nearby stars has begun.
\end{abstract}

Keywords: adaptive optics, coronagraphy, exoplanets, extrasolar planets, instrumentation

\section{INTRODUCTION}

For the first time in history, scientists can image the environments of nearby stars on scales approaching that of our solar system. New classes of astrophysical objects have been discovered including circumstellar debris disks, and brown dwarfs. This heralds a new era of research into comparative exoplanetary science. These discoveries have galvanized intense public interest and have led to profound new insights into the formation and evolution of planetary systems such as our own. Among the key enabling technologies are adaptive optics (AO) and coronagraphy, which deliver the high contrast necessary for the discovery and characterization of faint stellar companions and circumstellar disks in the solar neighborhood.

The Lyot Project is a multifaceted research and development program based at the American Museum of Natural History. The project is designed to advance the techniques and science required for exoplanetary science, with a particular emphasis on the combination of $\mathrm{AO}$ and coronagraphy. The project is named to honor Bernard Ferdinand Lyot (1897-1952), inventor of the solar coronagraph, an instrument which on 12 July 1931 acquired the first images of the Sun's corona without a solar eclipse. ${ }^{1}$ Lyot conducted spectroscopic study of the corona through his coronagraph and even made the first movie of solar prominences with it. ${ }^{2}$

The Lyot Project's initial goal has now been achieved: the construction, deployment and use of the world's first coronagraph optimized for, and operating at, the diffraction limit of a telescope (a direct extension, as described by Sivaramakrishnan et al. in $2001^{3}$, of Lyot's original idea ${ }^{1}$ to its physical limit in application to imaging the regions around stars other than the Sun). The project uses this instrument not only for a survey of nearby stars, but also as a basis for the investigation, development and implementation of advanced concepts in extremely high-contrast imaging.

In our efforts to optimize stellar coronagraphy, we use the US Air Force's Advanced Electro-Optical System (AEOS). Among the AO systems available to astronomers, AEOS is unique because it delivers the highest order wavefront correction ${ }^{4}$. Our program, to build and deploy an AO optimized coronagraph, exploits the full astronomical

* bro@amnh.org; phone 1212313 7921; fax 1212769 5007; lyot.org 
potential of AEOS for high-contrast imaging, and represents a critical step toward the long-term goal of directly imaging and studying exoplanets.

Below we present a brief overview of the basic concepts in this line of research, the opto-mechanical design of the Lyot Project coronagraph, initial results from the first-light observations, and a description of the Project's future research.

\section{AO CORONAGRAPHY}

$\mathrm{AO}$ increases angular resolution and more importantly contrast, so that a faint object can be detected close to a bright one that would otherwise overwhelm it. Coronagraphy artificially suppresses the light of a bright star. To quantify the astronomical utility of an AO coronagraph we consider two factors: 1) the degree of contrast enhancement;

2) the radii at which enhanced contrast is achieved.

1. Consider the contrast improvement when an AO system is used to observe two stars separated by an angle smaller than the seeing disk but larger than the Airy disk. Suppose $S_{\mathrm{AO}}$ and $S_{\mathrm{see}}$ are the Strehl ratios in corrected and uncorrected images. A useful measure of the contrast improvement, $\eta$, is the product of two factors: the ratio $S_{\mathrm{AO}} / S_{\text {see, }}$, due to sharper images, and the factor, $1 /\left(1-S_{\mathrm{AO}}\right)$, due to the amount of light removed by AO from the seeing disk. Hence,

$$
\eta=\frac{S_{A O}}{S_{\text {see }}} \frac{1}{\left(1-S_{A O}\right)}
$$

For low-order systems (e.g., Palomar ${ }^{5}$ or Lick), $S_{\mathrm{AO}} \sim 0.5$ and $S_{\text {see }} \sim 0.03$, and $\eta \sim 30$. For low Strehls image sharpness is the dominant factor. But as $S_{\mathrm{AO}} \rightarrow 1$ large improvements accrue as progressively more light is removed from the seeing disk. The AEOS system has enormous astronomical potential because it delivers $S_{\mathrm{AO}} \approx$ 0.89 in the near-IR at $1.6 \mu \mathrm{m}$ (see Section 5 below) and gives $\eta \geq 300$. This advantage diminishes at shorter wavelengths. For example, at $I$-band $(0.9 \mu \mathrm{m})$ the Strehl ratio drops to 0.25 and the corresponding contrast enhancement is a factor of thirty less. This steep wavelength dependence, together with our desire to find cool (effective temperature, $T_{\text {eff }} \leq 1000 \mathrm{~K}$ ) companions drives the requirement to observe in the near IR. ${ }^{\ddagger}$

Simulated AO Corongraph Point Spread Functions

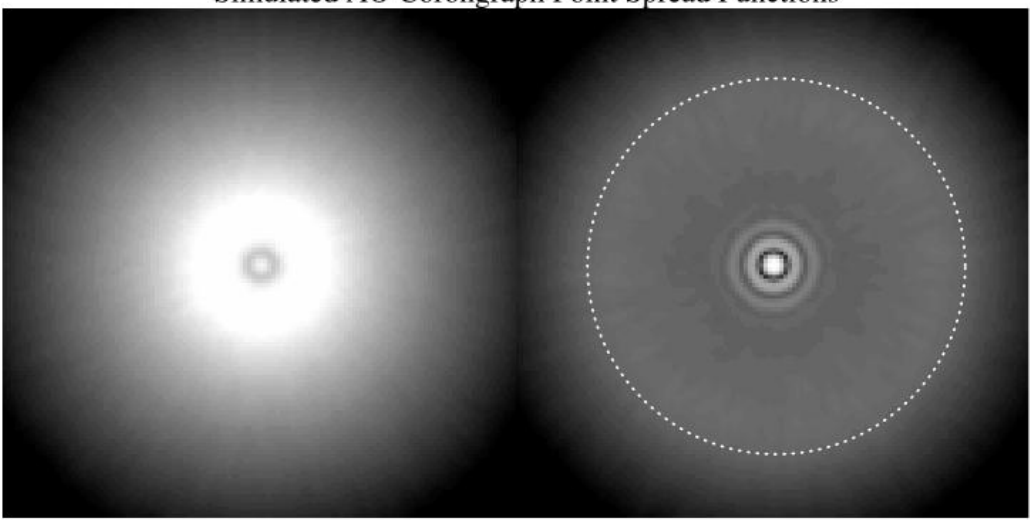

Fig. 1 Comparison of low- and high-order coronagraphic AO PSFs simulated in white light through the H-band on a 3.6-m telescope over a $4 \times 4$ arcsec field of view. On the left (a) is a simulation with $\mathrm{N}_{\mathrm{act}}=16$, on the right (b) $\mathrm{N}_{\text {act }}=34$ (comparable to AEOS). The clearing of the halo is obvious within the dotted circle on the right hand image. This circle has a radius of approximately 1.5 arcseconds at this wavelength, which is the angle $\theta_{\mathrm{AO}}=\mathrm{N}_{\mathrm{act}} \lambda / 2 D$. $^{9}$

(a) 16 Actuators across the Pupil

(b) 34 Actuators across the Pupil

2. The full improvement in contrast exists only within a radial distance from the star, $\theta_{\mathrm{AO}}$, which is determined by the design of the $\mathrm{AO}$ system. ${ }^{3}$ AO correction can be thought of as a high-pass filter acting on spatial phase variations of incoming wavefronts. The AO system's spatial frequency cutoff is $k_{\mathrm{AO}}=N_{\mathrm{act}} / 2 D$, where $N_{\text {act }}$ is the number of deformable mirror actuators projected linearly across the primary of diameter $D$. The electric field at the image and pupil planes are a Fourier pair, so $k_{\mathrm{AO}}$ corresponds to an angle $\theta_{\mathrm{AO}}=\mathrm{N}_{\text {act }} \lambda / 2 D$ on the sky at wavelength $\lambda$. Thus AO only improves the point spread function (PSF) within a radius $\theta_{\mathrm{AO}}$ (within the dotted circle in Fig. 1(b)). ${ }^{*}$ The

\footnotetext{
¥ Please note: this ad hoc analysis is meant to provide an order of magnitude estimate. A fuller understanding requires using the true structure and range of spatial scales present in the corrected wavefront. Strehl acts as a proxy for the rms wavefront error.

${ }^{*}$ This statement is true under the assumption that there is no residual image motion and that the system is focused.
} 
Lick 3-m, Palomar 5-m, and AEOS 3.63-m systems have 8, 16 and 34 actuators projected across their entrance pupil diameters. Thus, on these systems, AO improves a stellar image to distances of $4(0.4 \operatorname{arcsec}), 8(0.5 \operatorname{arcsec})$ and 17 (1.5 arcsec) diffraction widths respectively at $1.6 \mu \mathrm{m}$ (Fig. 1).

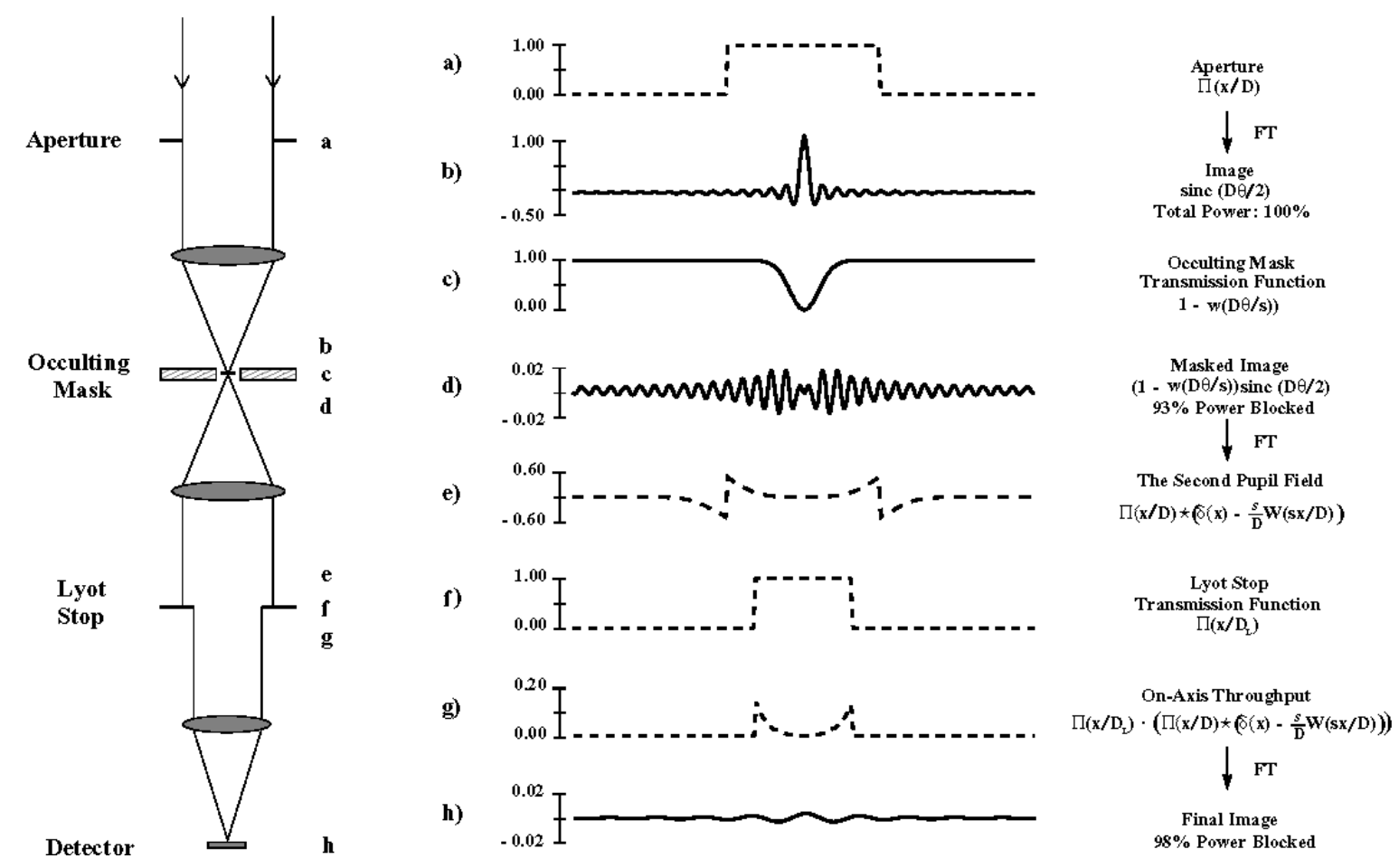

Fig. 2 Principles of coronagraphy. ${ }^{3}$ Panels (a)-(h) show the one-dimensional E-fields for an on-axis star and the transmission functions of the optical components. Comparison of the E-fields in image planes $(b$ and $d)$ before and after the focal plane mask (c) show the effects of the occulting disk. The diffraction pattern of the occulted star in the pupil plane (e) is masked by the Lyot stop (f) producing the spatially filtered E-field (g) of the final image (h). ${ }^{3}$

Exoplanetary science demands high contrast on angular scales corresponding to solar system dimensions (1-30 AU) for nearby stars $(5-30 \mathrm{pc})$ or within the seeing disk at radii of about 1 arcsec. AO alone cannot achieve the very high dynamic range necessary to find faint companions and circumstellar disks. This requires a coronagraph or other technique that removes the starlight. A coronagraph, ${ }^{1,3}$ consists of two stops placed in the reimaged focal and pupil planes (Fig. 2) respectively. The occulting mask (Fig. 2) is an opaque disk, optimally 3 to 9 times the size of the core of the stellar image. This mask blocks most of the on-axis starlight. The effect of this mask on (b) is shown in (d). After the occulting mask, the pupil is reimaged (e), where the remaining starlight is distributed as the Fourier transform of (d). This light is associated with the Airy rings of the image in a periodic spatial distribution; its periodicity is of the order of the telescope's angular resolution $(\lambda / D)$. Thus, this energy appears predominantly in an annulus at the edges of the pupil (e). The undersized Lyot stop (f) removes this light at the expense of some reduction of the telescope collecting area. In essence, the Lyot stop is a spatial filter that removes the high spatial frequency components of the electric field. The central obscuration scatters light into a similar bright ring around the inner edge of the pupil. The Lyot stop must mask this too, so that a small secondary (such as that on AEOS) is a great advantage. On AEOS an optimized coronagraph lets up to $34 \%$ more light through than a similar system on a typical telescope with a secondary diameter a third of that of the primary. The net result is that an on-axis source behind the aperture mask is suppressed by several orders of magnitude relative to an off-axis target.

In an optimized coronagraph ${ }^{3}$ the occulting spot must span at least four diffraction widths in diameter ( $\left.4 \lambda D\right)$. This results in little gain from using a coronagraph with AO systems where $N_{\text {act }}<8$, since the occulting spot occludes most of the improved image area: At Palomar, with a $5 \lambda / D(0.34$ arcsec at $1.6 \mu \mathrm{m})$ radius spot, an improved image annulus only $3 \lambda D$ wide $(0.20$ arcsec) surrounds the spot. For AEOS the image is corrected out to $1.5 \operatorname{arcsec}($ at $1.6 \mu \mathrm{m})$. Thus the light from the central star is suppressed from the edge of the stop at 0.2 to 1.5 arcsec beyond which AO stops 
improving the PSF. The consequence is that an order of magnitude more stars can be searched on solar-system scales for companions and disks.

These arguments strongly suggest the tremendous potential of a coronagraph designed to exploit AEOS or any AO system with a large $\theta_{\mathrm{AO}}$. Large gains in scientific results will follow the deployment of such instruments.

\section{LYOT PROJECT CORONAGRAPH CAPABILITIES}

To show that a high-order AO coronagraph provides a unique and dramatic improvement over other facilities, and opens an entirely new parameter space in the direct detection of faint companions of nearby stars, we have simulated the performance of the AEOS adaptive optics system with a coronagraph. These simulations include detailed descriptions of atmospherically disturbed wavefronts, the telescope optics without spiders, a Shack-Hartmann wavefront sensor, a square geometry deformable mirror, including the five actuators which are inactive in the AEOS beam, the precise sizing and shape of the coronagraph's focal and pupil plane masks, and finally detector characteristics, including readnoise, sky background and quantum efficiency. ${ }^{3}$
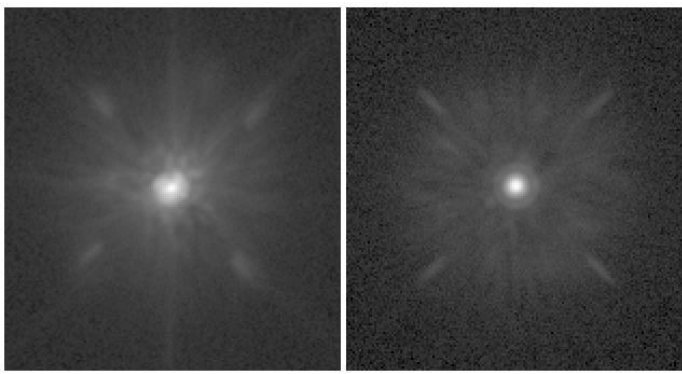

Fig. 3 Real AEOS I-band non-coronagraphic image on the left, with simulated image on the right. The figure reveals the integrity of our simulations. The simulation differs from the real data by less than $5 \%$ at all locations in the images. The differences are due to a residual tilt error in AEOS and CCD bleeding in the real data, as well as lower AO performance than expected ( $\mathrm{S} \sim 25 \%$ instead of the $40 \%$ in the simulations with the AO system operating perfectly). These discrepancies are understood (see Section 5).

Clearly we are attempting to simulate a fairly complicated system, with almost 20 optical surfaces. Thus it is critical to establish how meaningful these simulations are. We have compared them with actual, non-coronagraphic, AEOS I-band images (Fig. 3). (In the results section, our first real images are presented.) In this test case, we run the simulations in the $I$-band without the coronagraph, to model the facility VisIM visible imager camera. Our simulations reproduce the AEOS PSF shape with a precision of better than 5\% out to 3.5 arcsec separation from the stellar locus. Thus, we have accurately included all effects that are important for the science discussed here. The halo-clearing effect of the large number of actuators (Fig. 1) is the critical element of the PSF that we seek to exploit. This effect is two orders of magnitude larger than the differences between our simulations and actual AEOS data.

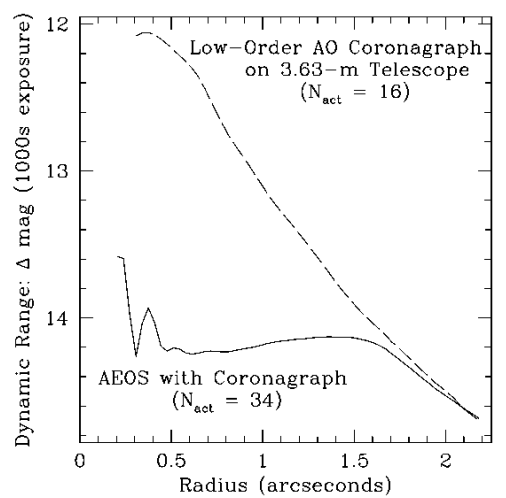

Fig. 4 Dynamic range for a $1000 \mathrm{~s}$ exposure with the coronagraph. Dynamic range is the difference in magnitude between the central star and a point source detected at the 5- $\sigma$ level. The detection limits are for the infrared H-band. ${ }^{9}$ A detailed discussion of how to extract curves such as these from data is presented in Oppenheimer et al. 2001. ${ }^{10}$

From the simulations of the AEOS coronagraph, we can determine the 5- $\sigma$ detection limits for faint point sources injected artificially into the AEOS coronagraphic PSF (Fig. 1(b)). The detection limits are completely determined by the residual light of the star (including speckle noise and photon-shot noise) and are expressed here in terms of dynamic range, the ratio of the flux of the faint companion to that of the bright, occulted primary star. Fig. 4 shows the simulated dynamic range as a function of angular separation from the primary star for an image of $1000 \mathrm{~s}$ exposure with the new instrument. The meaning of dynamic range here is such that in $1000 \mathrm{~s}$ we can detect a point source up to 14.2 magnitudes $\left(2 \times 10^{-6}\right)$ fainter than the primary star within 1.5 arcsec. Acoording to this, the Lyot Project coronagraph is more sensitive than the existing Palomar AO coronagraph by more than two magnitudes at 0.6 arcsec. Fig. 4 also shows 
the same curve for a theoretical lower-order AO coronagraph on a similar 3.63-m telescope. Beyond 1.5 arcsec, AEOS and the lower-order systems are roughly matched in sensitivity. This sensitivity is also about 7 magnitudes better than the Hubble Space Telescope's WFPC2 survey for faint companions of nearby stars within 1.5 arcsec of the stars. ${ }^{6}$

\section{OPTOMECHANICAL DESIGN}

The coronagraph is a visiting instrument which is installed in Coude Room 6 at AEOS. A $10.54 \mathrm{~cm}$ collimated beam from the AO system enters the Coude room through a beam port on the wall. The coronagraph processes the light and feeds an f/71 beam to the IR science camera, Kermit, built in Hawaii by Kuhn's group. The 32-channel readout electronics based on a Leach Gen 2 controller were developed at UC-Berkeley by Graham's group. The coronagraph is designed to be highly flexible with several pupil and focal planes providing locations for optical stops.

Fig. 5 shows the optical design, from overhead with all of the optical elements labeled. An enclosure (whose black frame is visible in Fig. 6) was fitted around the entire breadboard to minimize air currents in the optical path. The design has the beam 6.00 inches above the breadboard surface or 8.4 inches above Room 6's optical bench surface. A 2.4 inch thick, $4 \times 4$ foot breadboard supports the entire instrument, except for the dewar. The table at AEOS is $12 \times 4$ feet. Its surface can be positioned at 37.60 to 38.60 inches above the room's floor, which places the beam at a height of between 8 and 8.75 inches above the table surface. The coronagraph requires the beam to be near the middle of this range on the first optic. Tilts within 2 degrees in the input beam can be compensated by tilts in the first optic (BCM in Fig. 5). The optical bench floats during observations. The coronagraph optical train consists of the following pieces, all shown in Fig. 5. Surface figures are quoted below as peak-to-valley measurements at HeNe laser light $(\lambda=633 \mathrm{~nm})$.

The first optic (Beam Capture Flat or BCM) serves to capture the beam into the subsequently aligned path. BCM is at the reimaged telescope pupil. The optic is a 6 inch Zerodur $\lambda / 20$ flat in a precision gimbal mount. Placing $\mathrm{BCM}$ at the pupil plane is partly in anticipation of a possible upgrade of this instrument which would include a deformable mirror in the $\mathrm{BCM}$ location.

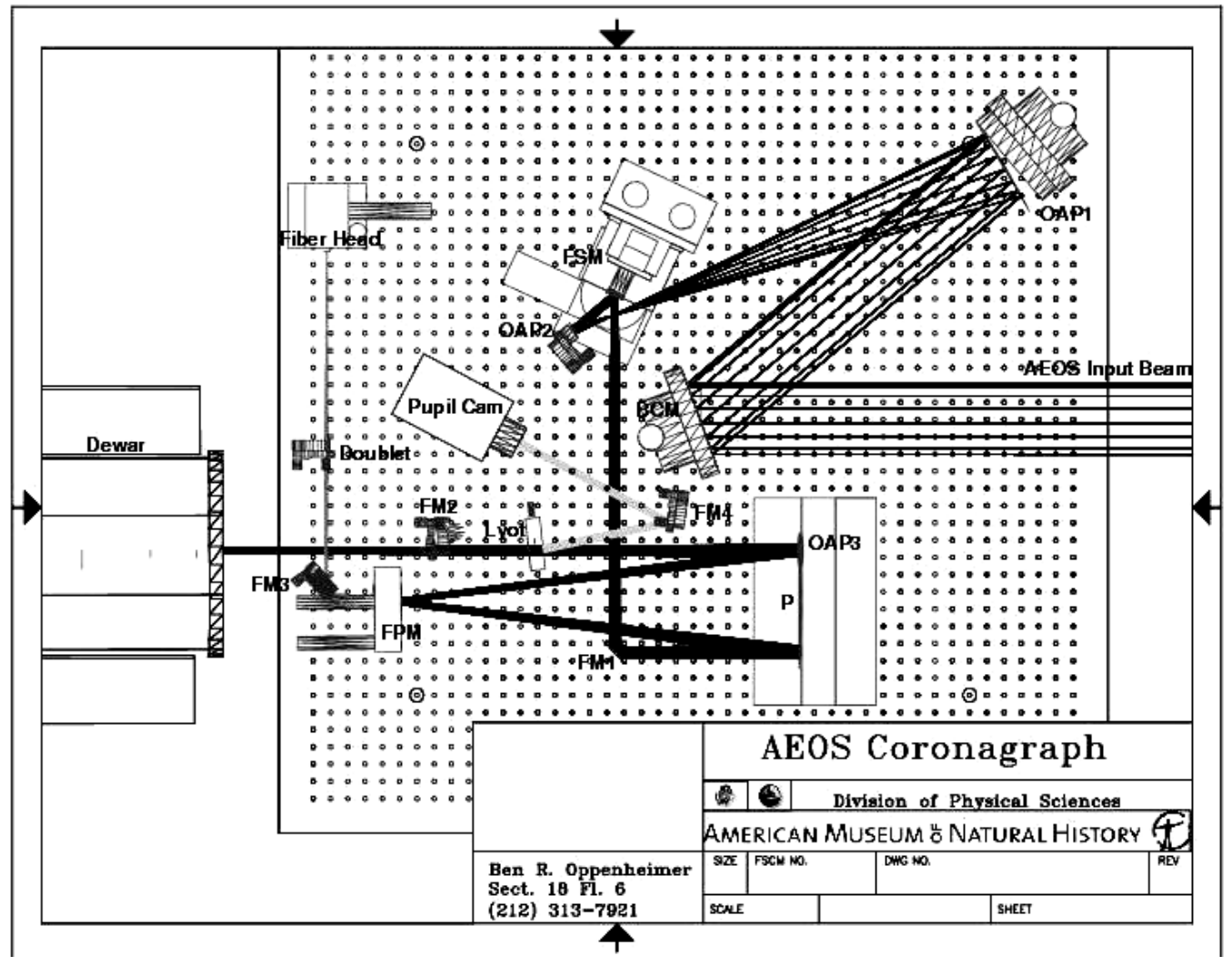

Fig. 5. Top-down view of optics design with components labeled 
The beam is compressed by a factor of ten to a $10.48 \mathrm{~mm}$ diameter by two off-axis parabolae (OAP1 and OAP2). Beam compression is needed in order to permit the fast $(1 \mathrm{kHz})$ tip/tilt system to be constructed at low cost. OAP1 is a 6 inch metal $\lambda / 20$ optic with a $600 \mathrm{~mm}$ focal length, situated $200 \mathrm{~mm}$ off axis (giving an 18.42 degree angle to the beam). The center of the optic is $200 \mathrm{~mm}$ off-axis. OAP2 is a custom, $25.4 \mathrm{~mm}$ diamond-turned aluminum mirror with a gold over nickel coating. It has a $60 \mathrm{~mm}$ focal length and is $20 \mathrm{~mm}$ off-axis. This compensates aberrations in OAP1.

OAP2 reimages the pupil plane about $60 \mathrm{~mm}$ after reflection. The Fast Steering Mirror (FSM) is situated $2 \mathrm{~cm}$ further along the beam path. Ideally the tip/tilt correction should be done exactly in the pupil plane to prevent any motion of the pupil image itself. In this case though, let us examine the expected pupil image motion due to the movement of FSM. 0.1 arcsec of tip/tilt on the sky corresponds to 36.3 arcsec at the FSM (magnification is 363 from the primary to the FSM) or $175 \mu \mathrm{rad}$. If we allow a pupil misalignment of $0.1 \%$ of a $10 \mathrm{~mm}$ beam $(10 \mu \mathrm{m})$, then $10 \mu \mathrm{m}$ / $175 \mu$ rad gives $5.7 \mathrm{~cm}$, which is the furthest the FSM can be situated from the pupil plane under this constraint. In truth, pupil misalignment up to a few percent of the beam diameter is permissible before substantial losses in dynamic range in the final image accrue. ${ }^{3}$ Moving the FSM $2 \mathrm{~cm}$ past the pupil image results in substantial improvements in the clearance between FSM's mount and the beam, although it permits pupil motion of up to $0.03 \%$ of the beam diameter.

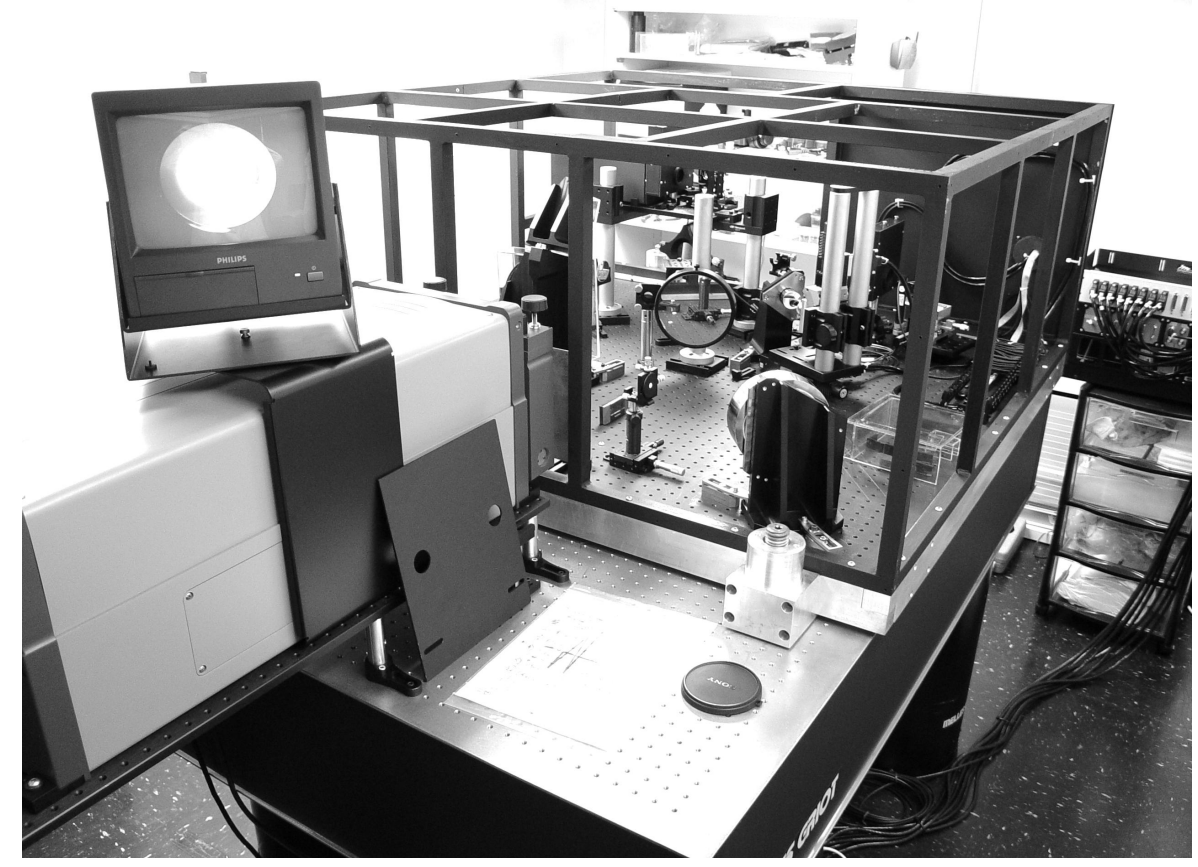

Fig. 6. Photograph of the coronagraph after initial alignment in the AMNH Astrophysics Lab's clean room. To the left is the Zygo interferomter used to simulate the AEOS beam to enable full testing in the lab prior to shipment to Maui. The residual wave front error through the entire system was $32 \mathrm{~nm} \mathrm{rms,98.7 \%} \mathrm{Strehl} \mathrm{in} \mathrm{the} \mathrm{H-band.} \mathrm{After} \mathrm{shipping} \mathrm{to} \mathrm{Maui,}$ the wave front error was measured at $29 \mathrm{~nm}$. The alignment technique will be presented in a subsequent publication, currently in preparation. None of the optics moved, nor was any of the alignment work compromised during shipping.

FSM is a $25.4 \mathrm{~mm} \lambda / 20$ Zerodur flat attached to a $25 \mathrm{~mm}$ aluminum flexure mount activated by a set of 4 PZT stacks. The mirror reflects the beam through a 50 degree angle ( 25 degree angle of incidence). The mount is attached to a spacing plate, which bolts into the faceplate of a goniometric cradle controlled with a stepper motor. This arrangement places the axis of rotation of the cradle at the center of the optic. The cradle is attached through a set of posts and thick plates to a rotation stage, also controlled with a stepper motor.

The PZT stacks can move the mirror $\pm 1 \mathrm{mrad}( \pm 0.57$ arcsec on the sky) at update rates over $1 \mathrm{kHz}$. The stacks, including the mount and a slightly larger mirror than the one we are using, have a resonant frequency of $2.4 \mathrm{kHz}$. Our electronics are capable of placing the star with an accuracy of $28 \mu$ as $(0.12$ nanorad) as measured on the sky (about 50 nanorad tilt error on the FSM). This substantially compensates the residual tilt error on the AEOS beam, currently measured at up to 200 milliarcsec. The system operates routinely at $1 \mathrm{kHz}$ update rates.

The two other rotation stages are used for placing the star on the coronagraph's focal plane mask. These motors will be inactive when the tip/tilt loop is operating, although they can be used to offload the PZT stacks. The rotation 
stages have a precision of $3.6 \operatorname{arcsec}$ or $17 \mu \mathrm{rad}$ at the FSM optic. This translates to 10 mas on the sky. For reference, the focal plane masks are 275 to 500 mas in diameter (as projected on the sky).

Mounting the PZT stack on the heavy goniometric cradle and rotation stage ensures mechanical stability. The mount is over 30 times as massive as the active PZT stage.

Fold Mirror 1 (FM1) simply folds the beam. The current design has two fold mirrors in the science beam, necessary to keep the instrument within the spatial constraints of Room 6. FM1 is a 1 inch $\lambda / 20$ Zerodur flat mounted in a post-raised gimbal mount.

The Parabola (P) on the lower right side of Fig. 5 serves as the heart of a pseudo-Offner relay that reimages the star at the focal plane mask and the pupil at the Lyot stop. $\mathrm{P}$ is 8 inches in diameter (the beam incident on the mirror is contained within a $140 \mathrm{~mm}$ circle centered at P's center). P has a focal length of $600 \mathrm{~mm}$. Surface accuracy for this optic is less stringent since the beam uses only a small region on opposite sides of the optical axis $(\lambda / 10)$. The $600 \mathrm{~mm}$ focal length provides an $\mathrm{f} / 57.25$ beam to the image mask.

The Focal Plane Mask (FPM, a. k. a. occulting mask) sits at the focus of P. The optical design provides perfect onaxis imaging at FPM, required for an optimized, diffraction limited coronagraph. The focal plane mask is a diamond turned flat mirror 2 inches in diameter. A hole is drilled through the center of the optic and beveled on the back side. The hole, ranging from 275 to $500 \mu \mathrm{m}$ in diameter, serves as the occulting mask. The starlight that passes through the hole is used to close the tip/tilt control loop. The f/57.25 beam results in a plate scale on the FPM of $1.0 \mathrm{mas} / \mu \mathrm{m}$. This large $\mathrm{f} / \#$ is necessary to avoid extremely expensive micromachining of these optics. Four FPMs have been made with holes to permit optimal occultation at all observing wavelengths. These parts posed significant problems for the manufacturer (Axsys Technologies). Trials using glass were unsuccessful due to chipping. A prototype mask made of diamond turned steel with a polished nickle coating covered with a gold optical surface revealed significant inhomogeneities around the hole edge. However, the best solution was to precision machine nickel which was then polished and coated with gold. The holes had deviations from circularity of less than $2 \mu \mathrm{m}$ and edges sharper than this as measured with an optical microscope. Examples of the steel and nickel mask holes are shown in Fig. 7.
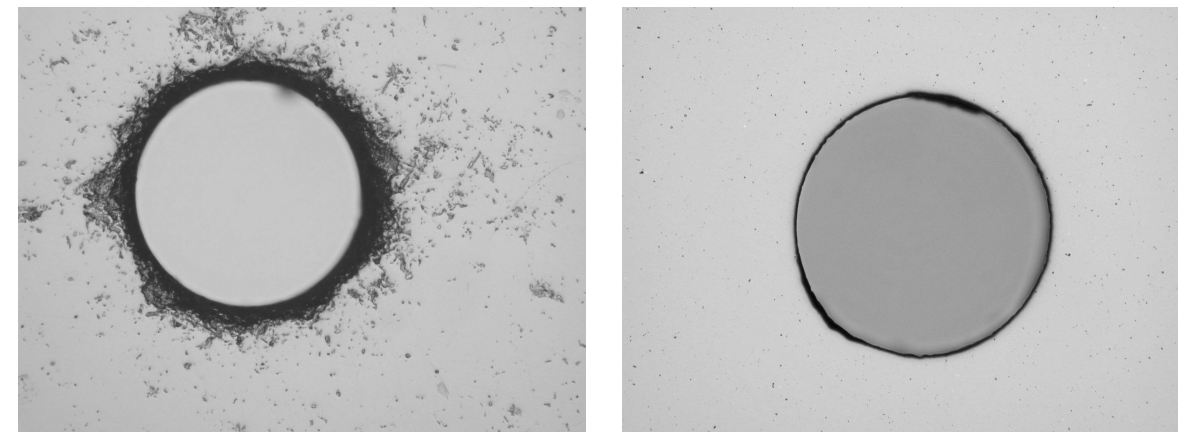

Fig. 5. Optical micrographs of the hole that forms the $334 \mu \mathrm{m}$ diameter H-band focal plane mask. On the left is the prototype mask made with diamond-turned steel coated with nickel. The nickel was polished to optical quality and coated with gold. On the right is a pure nickel mask polished and coated with gold. The mask on the right is used in routine observing mode.

The FPM is on a gimbal mount which is controlled with two DC servo motors. The servo motors can be used to precisely align the pupil with the Lyot stop. Furthermore, they can be operated to maintain pupil alignment whenever the telescope pupil is illuminated with a star. This is achieved with the pupil imaging camera (Pupil Cam) and a second control loop running along with the fast tip/tilt loop. Our science integrations will tend to be quite short (one to 300 seconds) due to the lack of an image rotator and the alt/az design of AEOS. In light of this and the fact that beam wander in AEOS is relatively slow, pupil alignment takes place between exposures.

The Lyot stop is located at a plane conjugate to the telescope pupil. The pupil is reimaged without aberration here. The Lyot stop itself is a metal mirror, reflective on the side facing P. The configuration shows it slightly tilted in Fig. 5. This is a 6 degree angle meant to reflect the light of the star from the regions of the beam that are obscured by the Lyot stop into Pupil Cam, situated above the FPM in Fig. 5. The camera is focused on the Lyot stop and permits measurement of Lyot stop-to-telescope pupil alignment. The Lyot stop is also mounted on a rotation stage so that the spiders can be matched to the telescope spiders.

FM2 is a gimbal mounted fold mirror, 1 inch in diameter made of $\lambda / 20$ Zerodur and coated with protected silver. This tilts the beam up away from the table and breadboard to feed the final focusing optic, OAP3, which is locaated directly above the P mirror. OAP3 is a diamond-turned custom Off-Axis Parabola, $750 \mathrm{~mm}$ focal length, 2 inch diameter, gold over nickel coating, $\lambda / 20$ surface, $270 \mathrm{~mm}$ off axis (measured to center of OAP3), mounted on a 
precision kinematic mount situated above the $\mathrm{P}$ mount. The beam, after impinging upon OAP3, is parallel to the bench surface and directly above the beam recollimated by P after the FPM.

This optic provides the final focus in front of the Dewar (the Kermit IR science camera). It is located one focal length away from the Lyot stop. The final beam entering the Dewar is F/71.34. This provides a plate scale of 14.73 mas/pixel at the detector. This design also transmits a $10 \mathrm{~mm}$ diameter unvignetted field of view (FOV) at the detector, while the actual detector spans $37 \mathrm{~mm}$, providing a viewable field 10.0 arcsec across. This small FOV allows dual or multiband imaging to be implemented in the future (Section 6).

The tip/tilt optics provide light for the control of the tip/tilt loop, which operates at an update frequency of $1 \mathrm{kHz}$. This necessitates an extremely fast detection method. Single photon counting modules (avalanche photodiodes) are presently the only suitable detectors. In our case, the limiting factor is the frame rate, not the number of photons available: Considering the throughput from the AEOS primary mirror to the APDs, a sufficient number of photons can be detected in $0.5 \mathrm{~ms}$ from stars brighter than $4^{\text {th }}$ magnitude in the 0.7 to $1 \mu \mathrm{m}$ range (the range of sensitivity for the APDs) to provide a centroid with a precision of $57 \mu$ as. (The signal-to-noise requirement here is 800 for an image size of 45 mas, which is the diffraction limit at the signal wavelength.) For a $7^{\text {th }}$ magnitude star, the centroiding error is approximately 400 uas. For the brightest stars, a neutral density filter is required to prevent APD saturation. A Gunn zband filter is also used to reduce the atmospheric dispersion difference between the star position detected by the APDs and the science band pass.

Four APDs serve as the tip/tilt detector, fed by a four-fiber bundle with a set of four lenslets on a single substrate positioned and aligned to the fiber heads. This "quad-cell" fiber pigtail is placed inside a fiber head micropositioner which provides $\mathrm{x}$ and $\mathrm{y}$ translation of the fiber head in the image plane. The image plane is formed with a doublet lens mounted behind the FPM (FM3 provides the needed clearance for the beam path). The motorized stage permits the system to track a star regardless of where the hole in FPM sits. The four fibers from the fiber pigtail are then connected directly into the APDs, which are housed in the electronics rack enclosure.

The doublet forms an image of the FPM hole at the lenslet in front of the quadrant fiber head. The doublet has a $150 \mathrm{~mm}$ focal length, giving an Airy spot diameter of about $150 \mu \mathrm{m}$ at the lenslet surface, where the quad-cell head is $280 \mu \mathrm{m}$ across.

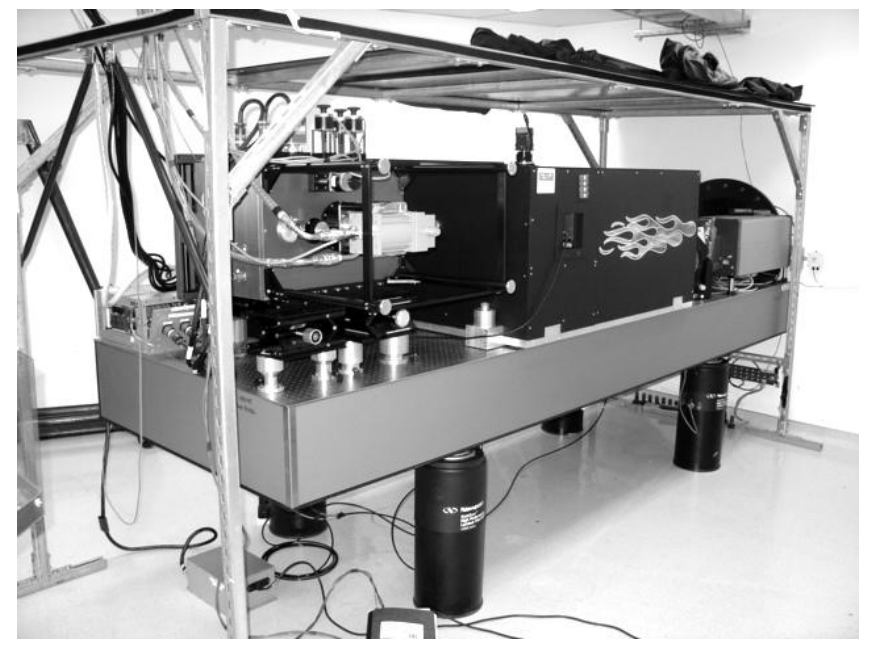

Fig. 6. The Lyot Project Coronagraph as installed in Room 6 at AEOS in March 2004. The Dewar on the left is the Kermit IR camera. The large black box with flames is the coronagraph. The grey box on the right is a Zygo interferometer used to align the coronagraph to the telescope beam. To do this, a theodolite is set up on the opposite side of the wall with the black circular beam port (just behind the Zygo in this picture). The theodolite is set to mimic the angle and location of the telescope beam. A mirror is placed at the entrance aperture of the coronagraph and autocolimated to the theodolite. By inserting a fold mirror, the Zygo beam can be autocollimated to this mirror as well, thereby making the Zygo beam identical in tilt and location to the telescope beam. This is done because the telescope alignment beam is extremely faint in Room 6, since it was not designed to serve the instruments in the AEOS experiment rooms. The control electronics are located in an adjacent room, where data are collected.

\section{CURRENT PROJECT STATUS}

The Kermit IR camera underwent first light observations using the engineering grade detector in April 2003. It functions well. In rather poor weather conditions (2" seeing in the optical), Kermit was able to make an image with a Strehl ratio of approximately $60 \%$ in the $\mathrm{H}$ band. This was particularly encouraging and seems to confirm the notion that a $90 \%$ Strehl will be achieved during median seeing conditions in the H-band.

The coronagraph, with assembly completed in New York at the American Museum of Natural History, underwent extensive testing after December 2003. It shipped to Maui on March 4, 2004, where it was installed in AEOS's Experiment Room 6. On 11 March 2004, the coronagraph was fed starlight from the AEOS telescope for the first time. 
After spending only 5 hours of telescope time to bring the whole system to a fully operational state, we observed the star 55 Cancri, a star with a known exoplanetary system (consisting of 3 planets, including a 4 Jupiter-mass object at an angular separation of about 4 arcseconds). This is a rather old star, so we did not expect to be able to detect any of the planets, but it was a good target for our initial observations. 55 Cancri was observed in the H-band with occulted and unocculted images taken. Total integration time was 11 minutes. We are still in the process of analyzing the data and have determined most of the basic steps of data reduction for this new type of optimized, "extreme" AO, coronagraphic data. In Fig. 9, we present two of the first exposures of this star, one with the star under the FPM and one unocculted. These data are extremely interesting. First, they demonstrate the predicted ${ }^{7}$ effect of speckle symmetry in a coronagraphic PSF. This effect is only visible when the input wavefront has a Strehl ratio above $85 \%$. Our unocculted images reveal a PSF with $90 \%$ Strehl, arguably the highest ever achieved in the H-band on any instrument.
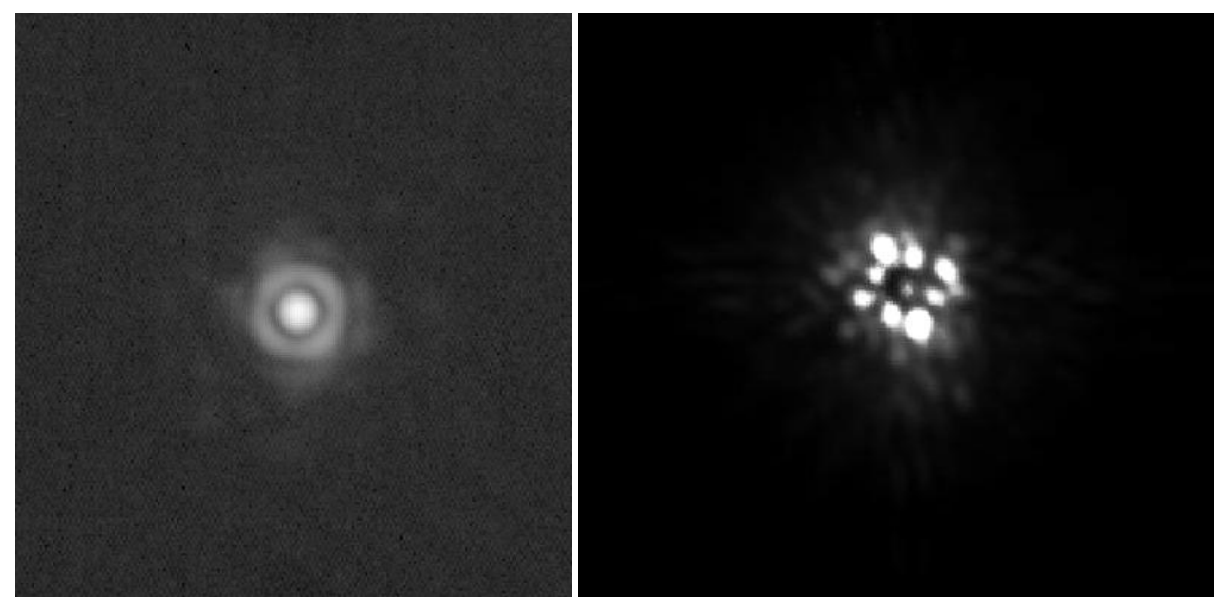

Fig. 9. Two first-light images of the star 55 Cancri. On the left: Unocculted 2 second image 2.5 arcsec on a side in the H-band. This image has a Strehl ratio of approximately $90 \%$. Right: oneminute coronagraphic exposure in the H-band, 5.0 arcseconds in width. Speckle symmetry is obvious. Greyscale is logarithmic.

The March 2004 data were acquired during only 11 hours of usable observing time, due to rather poor weather that ended Maui's long-term drought. These data have enabled us to begin to characterize the true performance of the coronagraph and the $\mathrm{AO}$ system.

In the comparison of I-band data with simulations (Fig. 3), we noted that the actual Strehl ratio was about $25 \%$ while simulations suggested that it should be $40 \%$. There are several reasons that the H-band coronagraphic data was so far superior and directly in line with the simulation predictions of image quality (89\%). First, the VisIM camera which formed the I-band images has a Strehl ratio of only $70 \%$. ${ }^{4}$ Second, the Lyot stop removes the outer portion of the telescope primary, and accordingly the outer portion of the deformable mirror. The AEOS AO system has slaved two subapertures of actuators on the outer edge of the deformable mirror. This means that the outer edge of the wavefront is not being corrected properly. Since the Lyot stop removes this, the final image is not susceptible to this issue. In addition there are five broken actuators on the deformable mirror. ${ }^{4}$ All but one are blocked by the Lyot stop.

Furthermore, while we are still trying to understand this new data, the halo clearing effect described in Section 2 appears to extend only to about a one arcsecond radius rather than the predicted 1.5 arcseconds. The deformable mirror is operated with only half of its available stroke because of fear that the broken actuators might either cause destruction of the mirror or separation of additional actuators. The effect of using only half stroke is that it requires two subapertures to correct a full stroke slope on the wavefront. In essence one can think of this as effectively increasing the inter-actuator spacing. That results in a somewhat smaller $\theta_{\mathrm{AO}}$. Further work on the data is necessary to fully understand it. We are in the process of calculating the achieved dynamic range from the system, but results were not ready prior to the due date of this manuscript.

\section{FUTURE RESEARCH}

The flexibility of the Lyot Project coronagraph's design makes it an ideal facility for testing novel coronagraphic techniques. Over the next year, while conducting our survey of nearby stars, we will implement and evaluate the following: an apodized pupil mask, three types of focal plane phase masks, and a dual band polarimetry experiment for increased sensitivity to circumstellar disks. We also intend to implement an integral field spectroscopic mode. This has been recently identified as a technique that can greatly reduce the speckle noise effect. ${ }^{8}$ 


\section{ACKNOWLEDGEMENTS}

The Lyot Project is based upon work supported by the National Science Foundation under Grant No. 0334916 and 0215793. The Lyot Project grateful acknowledges the support of the US Air Force and NSF in creating the AEOS/ATI opportunity that provides access to the AEOS telescope. Eighty percent of the funds for that program are provided by the US Air Force. The Lyot Project is also grateful to the Cordelia Corporation, the Vincent Astor Fund and two anonymous donors, who enabled the construction of the AMNH lab and initiated the project. Digby and Soummer are supported by Michelson Postdoctoral Fellowships. Perrin is supported by a Michelson Graduate Fellowship. Oppenheimer is partially supported by the American Museum of Natural History's Kalbfleisch Fund. The Lyot Project also recognizes the exemplary efforts of Mike Sweeney and his team at Axsys Technologies who were commissioned to build the custom optics and the focal and pupil plane optical stops. This work is based on observations made at the Maui Space Surveillance System operated by Detachment 15 of the U.S. Air Force Research Laboratory's Directed Energy Directorate. Sivaramakrishnan and Makidon acknowledge support from the Space Telescope Science Institute's Director's Discretionary Fund.

\section{REFERENCES}

1. Lyot, B., Study of the Solar Corona and Prominences without Eclipses, Monthly Notices of the Royal Astronomical Society, Vol. 99, 578-590, 1939.

2. D'Azambuja, L., L'ouvre de Bernard Lyot, L'Astronomie, Vol. 66, 267-277, 1952.

3. Sivaramakrishnan, A., Koresko, C. D., Makidon, R. B., Berkefeld, T., and Kuchner, M. J., Coronagraphy with High-Order Adaptive Optics, Astrophysical Journal, Vol. 552, 397-405, 2001.

4. Roberts, Lewis C., Jr. and Neyman, Christopher R., Characterization of the AEOS Adaptive Optics System, Publications of the Astronomical Society of the Pacific, Vol. 114, 1260-1266, 2002.

5. Oppenheimer, B. R., Dekany, R. G., Hayward, T. L., Brandl, B., Troy, M., and Bloemhof, E. E., Companion Detection Limits with Adaptive Optics Coronagraphy, Proceedings of SPIE, Vol. 4007, 899-905, 2000.

6. Schroeder, D. J. et al, A Search for Faint Companions to Nearby Stars Using the Wide Field Planetary Camera 2, Astronomical Journal, Vol. 119, 906-922, 2000.

7. Perrin, M. D., Sivaramakrishnan, A., Makidon, R. B., Oppenheimer, B. R., Graham, J. R., The Structure of HighStrehl Ratio Point Spread Functions, Astrophysical Journal, Vol. 596, 702-712, 2003.

8. Sparks, W. B., Ford, H. C., Imaging Specctroscopy for Extrasolar Planet Detection, Astrophysical Journal, Vol. 578, 543-564, 2002.

9. Oppenheimer, B. R., Sivaramakrishnan, A., Makidon, R. B., Imaging Exoplanets: The Role of Small Telescopes, in The Future of Small Telescopes In The New Millennium, T. Oswalt, ed., Vol. III, (Dordrecht: Kluwer), 2003.

10. Oppenheimer, B. R., Golimowski, D. A., Kulkarni, S. R., Matthews, K., Nakajima, T., Creech-Eakman, M., Durrance, S. T., Coronagraphic Survey for Companions of Stars within 8 Parsecs, Astronomical Journal, Vol. 121, 2189-2211, 2001. 